\title{
Three-bucket quadrature phase stepping in a shearing speckle interferometer
}

\author{
Peter A.A.M. Somers ${ }^{*}$, Nandini Bhattacharya \\ Optics Research Group, Delft University of Technology \\ Lorentzweg 1, NL-2628 CJ Delft, the Netherlands
}

\begin{abstract}
Phase stepping algorithms are mostly based on three or more interferograms that can either be acquired sequentially, involving some temporal phase stepping mechanism, or in parallel. When a phase step is applied between acquisitions, object phase changes may cause phase errors when calculating phase. A new system that allows the object to change in between arbitrary temporal phase steps is proposed. It comprises a relatively simple polarization based two-channel speckle interferometer that acquires two $\pi / 2$ phase stepped interferograms simultaneously with a single camera. This quadrature pair is phase stepped with a temporal phase stepping system that is also polarization based. Simulations and experimental results are presented that illustrate the improvements achieved with quadrature phase stepping compared to results obtained with the two-channel speckle interferometer operated without additional temporal phase stepping.
\end{abstract}

Keywords: speckle interferometry, polarization phase stepping, quadrature phase stepping

\section{INTRODUCTION}

Speckle interferometry is a useful tool for quantitative evaluation of deformation or shape, producing phase distributions that represent a given state of the object. Phase stepping methods ${ }^{1}$ are widely used to derive phase from speckle interference patterns. Acquiring a series of phase stepped speckle patterns involves either a sequential approach or a parallel one. Both methods have their own implementations in hardware. Sequential methods often use piezo-electric devices to adjust the optical path length of one of the interfering beams, parallel methods are based on multi-channel systems or on spatially phase stepping set-ups. Most parallel systems produce fixed phase steps. Polarization sensitive devices can take care of the appropriate optical path differences in each of the optical channels ${ }^{2}$, or even between adjacent pixels, for instance implemented as a micropolarizer array, as recently proposed. Other parallel phase stepping systems use tilted reference beams to obtain a fixed phase step between adjacent pixels. All parallel systems have in common that all phase stepped interferograms representing a specific state of the object at a certain moment in time are acquired simultaneously. This is an important advantage of such systems when measuring dynamic events. Parallel systems however can be complicated when more than a few optical channels are involved, make less efficient use of available light when spatial phase stepping is used, or rely on non-standard polarization sensitive masks that have to be aligned carefully to the CCD chip. Alignment is crucial as well for multi-channel systems ${ }^{3}$. Sequentially phase stepped systems must deal with generally unknown phase changes of the object in between phase steps, but their construction can be simple, independent of the number of phase steps. When using piezo-electric devices the calibration of the phase steps requires special attention, handling non-linearity, drift, and hysteresis of these devices. It is with these considerations in mind that a moderately simple 2-channel parallel system, with a fixed $\pi / 2$ phase $\operatorname{step}^{2,3,4}$ has been extended to a parallelsequential system, combining the advantages of parallel and sequential acquisition.

\section{ALGORITHM QUADRATURE PHASE STEPPING}

Two-bucket phase stepping is sufficient to perform quantitative interferometry. The algorithm which is based on taking one $\pi / 2$ phase stepped pair of interferograms before an event, and one pair after it, can be used to calculate phase

\footnotetext{
*P.A.A.M.Somers@TUDelft.nl; N.Bhattacharya@TUDelft.nl; phone +31 (0)15 27 84288; fax +31 (0)15 27 88105; http://www.optica.tn.tudelft.nl/

Speckle06: Speckles, From Grains to Flowers, edited by Pierre Slangen, Christine Cerruti, Proc. of SPIE Vol. 6341, 63411K, (2006) · 0277-786X/06/\$15 · doi: 10.1117/12.695376
} 
differences. Applying temporal phase unwrapping ${ }^{5}$, unwrapped results are obtained in real time, even for dynamic applications, since both $\pi / 2$ phase stepped interferograms are taken simultaneously. Even if the Eqs. $(1-4)$,

$$
\begin{array}{ll}
I_{1}=I_{B}+I_{M} \cos \left(\varphi_{1}\right), & I_{2}=I_{B}+I_{M} \sin \left(\varphi_{1}\right), \\
I_{3}=I_{B}+I_{M} \cos \left(\varphi_{2}\right), & I_{4}=I_{B}+I_{M} \sin \left(\varphi_{2}\right),
\end{array}
$$

from which Eq. (5) can be derived,

$$
\Delta \varphi=\varphi_{2}-\varphi_{1}=\pi / 2+2 \arctan \left[\frac{I_{1}-I_{4}}{I_{2}-I_{3}}\right]
$$

appear to be sufficient to solve phase difference, there are some conditions for which this is not the case: when both numerator and denominator in Eq. (5) are zero the 2-bucket algorithm breaks down ${ }^{6}$. Due to the presence of noise, results that are obtained from intensity values close to these breakdown points are also unreliable. Unreliable results are generally removed by spatial filtering, for instance by a filter following a spiral path ${ }^{4}$. Another possible solution is based on the involvement of data taken just before or after the unreliable intensity pair ${ }^{6}$. In the present work a more general solution is proposed. A $\pi / 2$ phase stepped data pair according to Eqs. (1-2) can be presented parametrically as a point on a circle, as shown in Fig. 1.

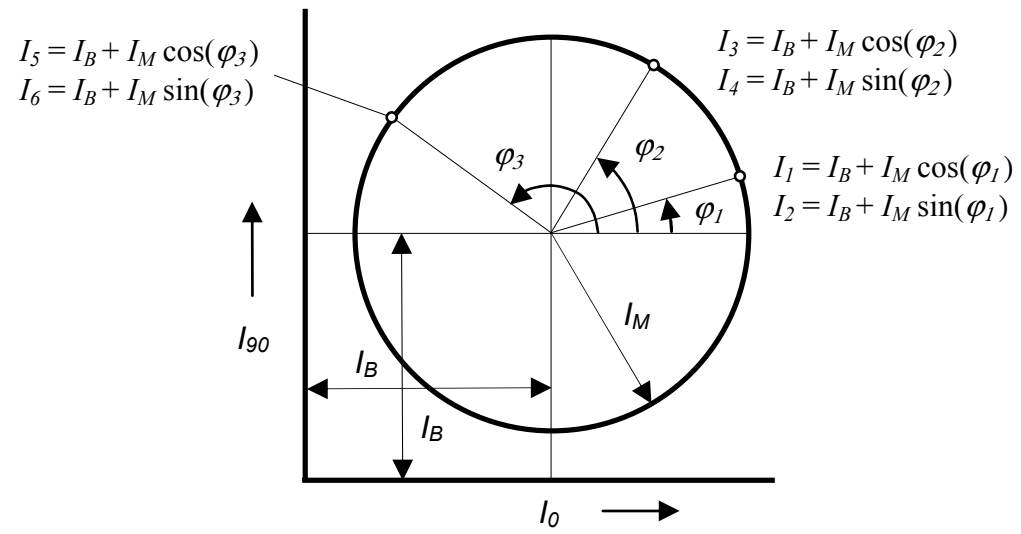

Fig. 1. Parametric presentation of three pairs of $\pi / 2$ spatially phase stepped interferograms. Temporal phase step between pairs is arbitrary. Intensity data belong to a single pixel

On the horizontal axis the cosine equation Eq. (1) is plotted against the sine Eq. (2) on the vertical axis. The radius of the circle is equal to the modulation $I_{M}$, the location of the centre is given by the value of the background $I_{B}$. There are three unknowns in Eqs. (1-2), so the unknown phase $\varphi_{1}$ cannot be resolved. In order to resolve phase or phase difference another quadrature data pair has to be acquired, adding one more variable, $\varphi_{2}$. This data pair is located on the same circle assuming that modulation and background remain the same. Phase difference $\Delta \varphi$ can be calculated with Eq. (5), but modulation and background can also be resolved ${ }^{6}$, restricted by the afore mentioned condition for breakdown. Breakdown is characterized in Fig. (1) by the condition that data pairs $I_{1}, I_{2}$ and $I_{3}, I_{4}$ are located symmetrically around the bisectrice running from the origin to the centre of the circle. The problem can be solved by acquiring a third quadrature pair, given by Eqs. (6-7), adding another variable $\varphi_{3}$ :

$$
I_{5}=I_{B}+I_{M} \cos \left(\varphi_{3}\right), \quad I_{6}=I_{B}+I_{M} \sin \left(\varphi_{3}\right) .
$$

This brings the number of unknowns to five, for which we have six equations. A solution can always be found if we have three different points on the circle, translating to the requirement that $\varphi_{2}$ and $\varphi_{3}$ should not be equal to $\varphi_{1}$ or differ by n $2 \pi$ from $\varphi_{1}$. Starting from unknown phase $\varphi_{1}$, phase changes $\varphi_{2}-\varphi_{1}$ and $\varphi_{3}-\varphi_{2}$ can now be considered as unknown sequential phase steps for quadrature pair $I_{1}, I_{2}$. It is apparent that apart from the requirement having three different points, these phase steps need not to be known to calculate phase $\varphi_{1}$. The object phase is allowed to change as well, again under the condition that three different positions on the circle are obtained. There is one additional condition: the 
sequential phase stepping system must be capable to return to its starting position when $\varphi_{1}$ was measured, or to a known position related to that reference position.

Instead of using analytical expressions derived from the six equations, calculations can be made based on geometrical properties of the circle. We have used Heron's formula to calculate the area of the triangle formed by the three points on the circle, and thereby the radius of the circle, equal to the modulation. By constructing a fourth point on the circle opposite to one of the three, a cross-section of the circle can be obtained, and the location of its centre. Having resolved background, phase can now be calculated for any of the three points on the circle using Eq. (8):

$$
\varphi_{1}=\arctan \left[\frac{I_{2}-I_{B}}{I_{1}-I_{B}}\right]
$$

When an arctangent lookup table for $\varphi$ is used these calculations don't require any tri-goniometric operations, which is an advantage for real time operation. After completion of the calculations another set of three quadrature pairs can be acquired to represent the state of the object after the event.

\section{POLARIZATION PHASE STEPPING SYSTEM}

Both the sequential and the parallel phase stepping sub-systems have been implemented in a shearing speckle interferometer using polarization sensitive optics (Fig. 2). The parallel system is based on the use of a polarization plane rotator, acting on two counter-rotating circularly polarized beams. The rotator is manufactured for a rotation of $\pi / 4$, taking care of a fixed phase step of $\pi / 2$. The parallel phase stepping mechanism has been described in more detail elsewhere ${ }^{2}$. The main components of the interferometer are the shearing section, the branch splitter and the combining unit. The two $\pi / 2$ phase-stepped images are projected side by side onto the same CCD, where they are acquired simultaneously. The temporal phase stepping mechanism is located in front of a polarizing beam splitting cube, which is the main component of the shearing section. Instead of a piezo-electric mirror that could be positioned in the shearing section to apply displacements to mirror M2, an adjustable half wave plate followed by a fixed quarter wave plate oriented at $45^{\circ}$ is placed in front of the PBC. This set-up has been proposed earlier for heterodyne interferometry ${ }^{7}$ but can also be used for phase stepping. The $\lambda / 4$ plate at $45^{\circ}$ converts linear light with an arbitrary direction to elliptical light. Special cases are horizontal and vertical light that are converted to circular light. Since the main axis of the ellipse is at + or $-45^{\circ}$, light is evenly distributed by the PBC: reflected and transmitted light have the same intensities, irrespective of the polarization direction of incoming linear light. There is a phase difference however between reflected and transmitted light which depends on the polarization direction of the incoming light. That direction can be influenced by adjusting the orientation of the $\lambda / 2$ plate in front of the $\lambda / 4$ plate.

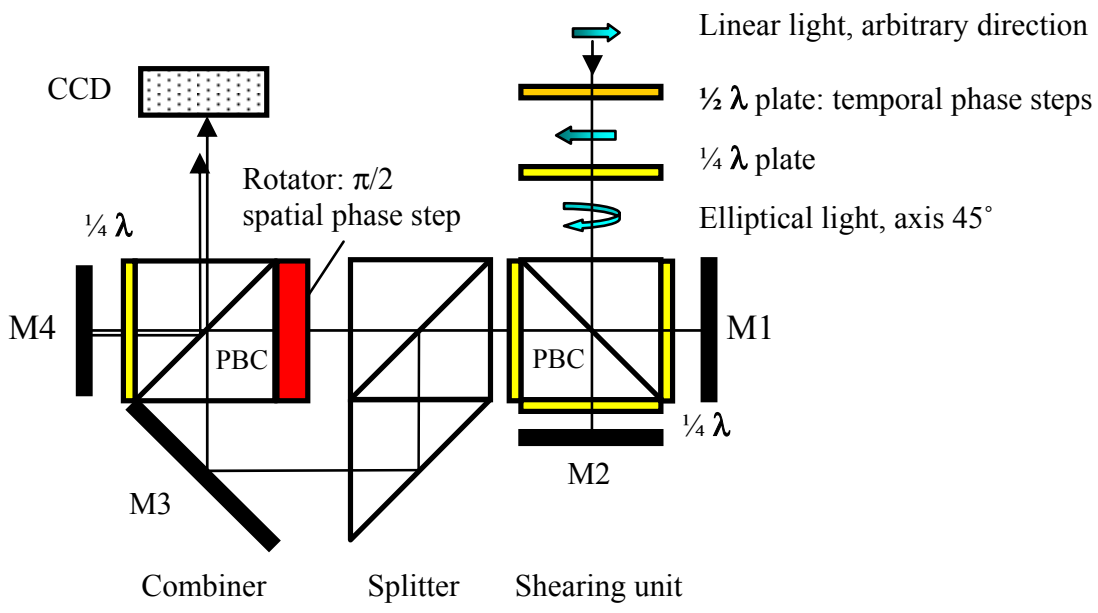

Fig. 2. Implementation of quadrature phase stepping system: quadrature pair is obtained by a fixed phase step of $\pi / 2$, provided by polarization plane rotator. Quadrature pair is stepped temporally by half wave plate. 
The properties of both parallel and sequential phase stepping systems can be described using Jones's calculus. Counterrotating circular light after passing a polarization plane rotator can be described by ${ }^{2}$ :

$$
V_{L C R o t}=\frac{1}{\sqrt{2}}\left[\begin{array}{l}
e^{-i \beta} \\
i e^{-i \beta}
\end{array}\right]=\frac{e^{-i \beta}}{\sqrt{2}}\left[\begin{array}{l}
1 \\
i
\end{array}\right], \text { and } V_{R C R o t}=\frac{1}{\sqrt{2}}\left[\begin{array}{l}
e^{i \beta} \\
-i e^{i \beta}
\end{array}\right]=\frac{e^{i \beta}}{\sqrt{2}}\left[\begin{array}{l}
1 \\
-i
\end{array}\right] \text {, }
$$

where $V_{\text {LCRot }}$ is left circular light, and $V_{R C R o t}$ is right circular light, both after passing the rotator, and $\beta$ is the rotation angle. Each of the circular beams has undergone a relative phase shift of $\beta$, one in positive, the other in negative direction, resulting in a total phase shift between the two of $2 \beta$. So a rotation angle of $\beta=\pi / 4$ results in a relative phase shift of $\pi / 2$ between the two circular beams. As a result the two intensity patterns on the CCD are allways in quadrature, irrespective of additional phase steps applied to both of them by the temporal phase stepping system.

The temporal system can be described as follows: light at arbitray angle $\alpha$ travelling through a quarter wave plate oriented at $45^{\circ}$ is given by

$$
M_{Q W P 45} V_{\alpha}=\frac{1}{2}\left[\begin{array}{cc}
1 & -i \\
-i & 1
\end{array}\right]\left[\begin{array}{c}
\cos \alpha \\
\sin \alpha
\end{array}\right]=\frac{1}{2}\left[\begin{array}{l}
\cos \alpha-i \sin \alpha \\
-i \cos \alpha+\sin \alpha
\end{array}\right]=\frac{1}{2}\left[\begin{array}{l}
e^{-i \alpha} \\
-i e^{i \alpha}
\end{array}\right]
$$

This is elliptical light. After passing the PBC in the shearing unit which acts as a horizontal polarizer for transmitted light, the Jones vector describing the light is given by:

$$
V_{H O R}=M_{H P} M_{Q W P 45} V_{\alpha}=\frac{1}{2}\left[\begin{array}{ll}
1 & 0 \\
0 & 0
\end{array}\right]\left[\begin{array}{l}
e^{-i \alpha} \\
-i e^{i \alpha}
\end{array}\right]=\frac{e^{-i \alpha}}{2}\left[\begin{array}{l}
1 \\
0
\end{array}\right]
$$

Similarly, the same elliptical light after being reflected by the PBC, acting now as vertical polarizer is given by:

$$
V_{V E R T}=\frac{1}{2}\left[\begin{array}{ll}
0 & 0 \\
0 & 1
\end{array}\right]\left[\begin{array}{l}
e^{-i \alpha} \\
-i e^{i \alpha}
\end{array}\right]=\frac{-i e^{i \alpha}}{2}\left[\begin{array}{l}
0 \\
1
\end{array}\right]
$$

Both beams have the same amplitude, their relative phase depends on the orientation $\alpha$ of the light entering the $\lambda / 4$ plate. The phase of both beams after the PBC changes with $\alpha$ in opposite directions as can be observed in Eqs. (12) and (13): the exponents $\alpha$ have different signs. Phase difference between the two beams is $2 \alpha$. The orientation $\alpha$ of linear light entering the $\lambda / 4$ plate can be adjusted by a $\lambda / 2$ plate in front of it; rotation of the $\lambda / 2$ plate over an angle of $\psi$ produces a change of orientation of $2 \psi$ of the incoming light, leading to a phase change of $4 \psi$ for the temporal phase stepping system.

\section{SIMULATIONS}

The behavior of the quadrature phase stepping method has been compared with the two- and four-bucket algorithms using simulations. A gradual phase change over $4 \pi$ has been assumed over the image, and intensity patterns for the three algorithms have been calculated. On top of ideal intensities different levels of random noise have been introduced, showing relative strength or weakness of a particular algorithm. In addition phase noise has been introduced to show that quadrature phase stepping is not sensitive to it, contrary to the two other algorithms. Fig. 3a shows three quadrature intensity patterns, used for the two-bucket and quadrature phase stepping system. Phase steps are at $0^{\circ}, 120^{\circ}$ and $240^{\circ}$. All patterns contain 4 full periods of fringes, representing the initial state of the object. Simulated interference patterns for the final state of the object contain 6 fringes. Only one pair has been generated for the final state: background calculated for the initial state has also been used for the final state. Fig. 3b shows a parametric plot of intensity data. Intensity noise level was $40 \%$. Fig. $3 \mathrm{c}$ shows the phase change over the image for the three algorithms, and horizontal cross-sections. The four-bucket algorithm shown here as a reference, is the less sensitive for intensity noise, the unmodified two-bucket algorithm shows large noise peaks around break-down positions. All three algorithms show false wrappings at the wrapping locations, caused by noise; the quadrature system shows a large improvement over the unmodified two-bucket system, but doesn't match the four-bucket algorithm for intensity noise. 


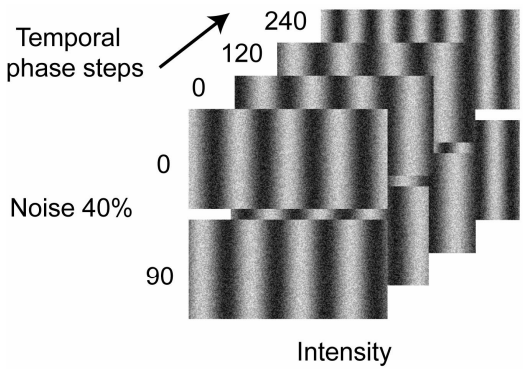

(a)

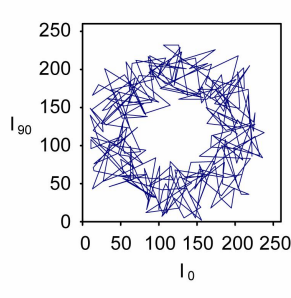

Parametric plot

(b)

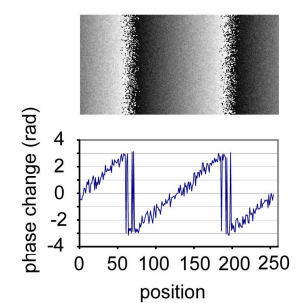

Four-bucket
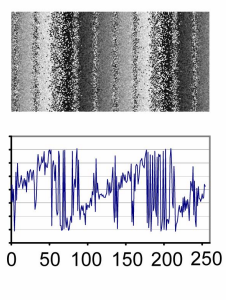

Two-bucket

(c)
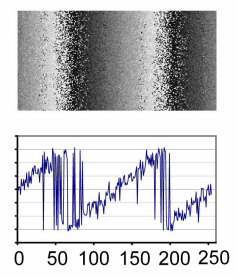

Three-bucket quadrature

Fig. 3. Simulated effects of $40 \%$ intensity noise for 3 algorithms. (a) Interferograms for the 3-bucket quadrature method. Three temporally phase stepped pairs before, and one after an event. Phase change is $4 \pi$. (b) Parametric plot of intensity pairs taken along a horizontal cross-section. (c) Phase change distributions and cross-sections for 4-bucket, 2-bucket, and 3-bucket quadrature methods.

In addition to the evaluation of intensity noise, simulations have been carried out to compare the sensitivity for phase noise of the quadrature phase stepping method with the four-bucket method. Again a gradual phase change of $4 \pi$ over the image width has been assumed, and four respectively six periods of fringes have been used to represent the state of the object before and after an event. For the first interferograms of both the four-bucket and the quadrature sets no phase noise has been assumed. Subsequent phase stepped interferograms, that is three interferograms for the four-bucket series, and two quadrature pairs for the quadrature series have been generated using a phase drift, varying in y-direction and increasing in x-direction. Quadrature pairs have been given the same drift locally, so these interferograms remain in quadrature. Results are shown in Fig. 4. Obviously, the results for the quadrature phase stepping method are not affected by phase drift, whereas results for the four-bucket method are seriously degraded.

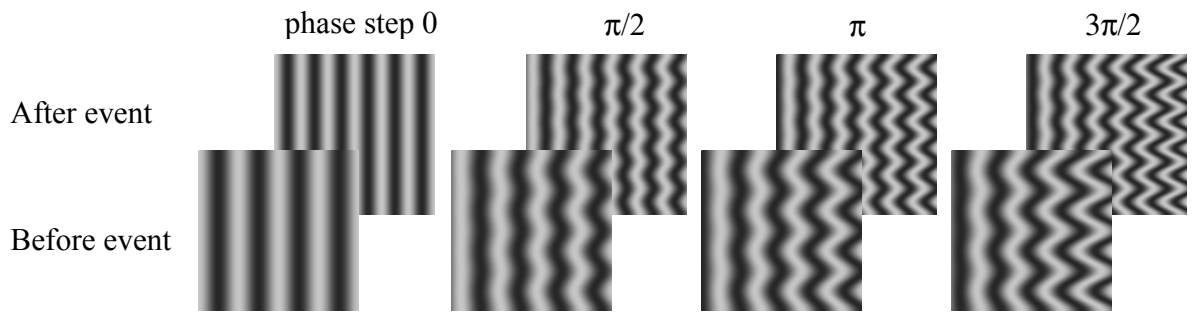

(a)

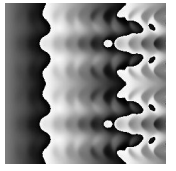

(b)

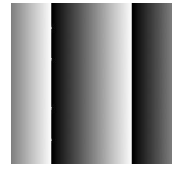

(c)

Fig. 4. Simulated effects of phase noise for two algorithms. (a) Simulated interferograms for four-bucket method. Four temporally phase stepped pairs before ( 4 fringes), and four after an event ( 6 fringes). Phase change is $4 \pi$. Similar patterns for quadrature method not shown. (b) Phase change distributions for four-bucket method. (c) Phase change distribution for three-bucket quadrature method.

\section{EXPERIMENTAL}

Some preliminary experiments to evaluate the performance of the new method compared to the standard two-bucket method have been carried out. A metallic structure consisting of a $0.4 \mathrm{~mm}$ Aluminum skin bonded to a honeycomb core and containing a circular debonded area of $24 \mathrm{~mm}$ has been thermally loaded with an incandescent lamp during three seconds. The object was followed while cooling down, acquiring interferograms with the interferometer described above. A series of three pairs of $\pi / 2$ phase stepped interferograms was taken, applying an additional temporal step of $2 \pi / 3$ between pairs. Phase has been calculated for each three-pair set, with the three-bucket quadrature phase stepping algorithm, using Eq. 8. Subsequently phase difference between the first pairs of each set has been calculated. The phase difference results have been accumulated, effectuating temporal phase unwrapping. For comparison with the two-bucket algorithm phase difference has been calculated directly for the first pairs of each of the three-pair sets, using Eq. (5). The second and third pair of each set have not been used for this calculation. The results are shown in Fig. 5. Fig. 5a shows the result for the two-bucket method, Fig. $5 \mathrm{c}$ for the quadrature method. For both methods a median filter was applied on selected pixels, operating along a spiral path $^{4}$, starting at the centre of the image. The selection was based on the detection of unreliable pixels, followed by a two-pass filter to remove noise spikes. Unreliable pixels were detected by setting a threshold on both modulation and background, that were calculated for the quadrature method. For the twobucket method the threshold was set on the difference between the two phase stepped interferograms, identifying low modulation and saturated pixels when the intensities are the same. Thresholds for both methods were set in such a way 
that the number of pixels involved in the filtering procedure was about the same. Fig. 5 shows the improvement obtained with the quadrature method: Fig.5d shows less noise than Fig. 5b.

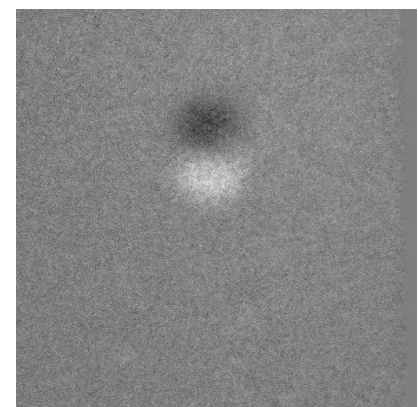

(a)

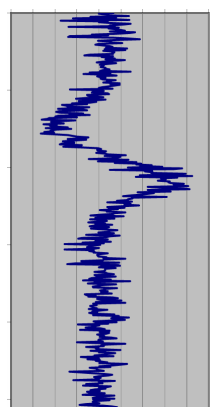

(b)

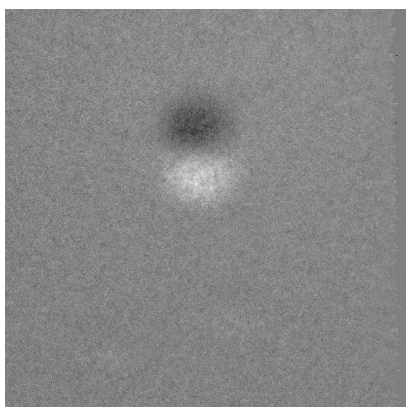

(c)

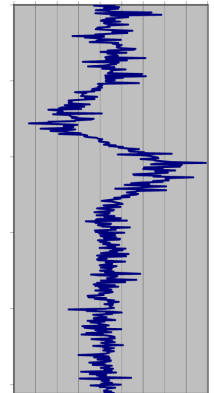

(d)

Fig. 5. Debonded area in a metallic honeycomb structure, defect size is $24 \mathrm{~mm}$. (a) Processed with the two-bucket phase stepping method. (b) Cross-section of (a). (c) Processed with three-bucket quadrature phase stepping. (d) Cross-section of (c).

\section{CONCLUSION}

A dual polarization based phase stepping system has been presented. The phase stepping system comprises a parallel system that uses a polarization plane rotator for a fixed phase step of $\pi / 2$ between two interferograms that are acquired simultaneously, and a sequential system based on a half-wave plate with adjustable orientation. The system allows quadrature phase stepping using three or more sequential phase steps. Sequential phase steps are arbitrary and need not to be known for the calculation of phase. The proposed method solves the break-down problem encountered with the unmodified two-bucket method. The new method shows its strength in particular in the presence of phase noise: the object or the medium between interferometer and object are allowed to change in between phase steps. The proposed method is therefore well adapted to dynamic applications and is very robust for quasi-static applications as well.

\section{ACKNOWLEDGMENTS}

This research was supported by the Technology Foundation STW - the Applied Science Division of NWO, and the Technology Programme of the Ministry of Economic Affairs. We thank Clothilde Zimmer, École Supérieure d'Optique, Institut d'Optique, Orsay, France, for her contribution to this research.

\section{REFERENCES}

1. B. V. Dorrio and J. L. Fernandez, "Phase-evaluation methods in whole-field optical measurement techniques," Measurement Science \& Technology, vol. 10, pp. R33-R55 (1999).

2. Peter A.A.M. Somers, Nandini Bhattacharya, "Polarization plane rotator used as a phase stepping device in a 2channel shearing speckle interferometer", SPIE conference "Optical Metrology 2005", Proceedings of SPIE, Vol. 5856, pp. 664-673, Wolfgang Osten, Christophe Gorecki, Erik Novak, eds. (2005)

3. Peter A.A.M. Somers, Nandini Bhattacharya, "Maintaining sub-pixel alignment for a single camera two-bucket shearing speckle interferometer." Journal of Optics A: Pure and Applied Optics, No 7, pp. S385-S391 (2005).

4. P.A.A.M. Somers and H. van Brug, "A single camera, dual image real-time-phase-stepped shearing speckle interferometer”, Fringe 2001, Proceedings, pp. 573-580, W. Osten, W. Jüptner, editors, Elsevier (2001).

5. J.M. Huntley and H. Saldner, "Temporal phase-unwrapping algorithm for automated interferogram analysis", Applied Optics Vol. 32, No 17, pp. 3047-3052 (1993).

6. P A.A.M. Somers, H. van Brug, and J. J.M. Braat, "A two-bucket phase-stepped shearing speckle interferometer: why does it work," Proceedings Speckle Metrology 2003, SPIE Vol. 4993, pp. 181-188, Kay Gastinger, Ole Jan Løkberg, Svein Winther, eds. (2003).

7. Gary E. Sommargren, "Up/down frequency shifter for optical heterodyne interferometry", J. Opt. Soc. Am. 65, pp. 960-961 (1975). 